A. MATSUI

KODAI MATH. J.

12 (1989), $5 \hat{\jmath}-71$

\title{
HIRZEBRUCH $L$-HOMOLOGY CLASSES AND THE INTERSECTION FORMULA
}

\author{
By AKINORI MATSUI
}

1. Introduction. In [7], Goresky and MacPherson introduced the signature for compact oriented $P L$-pseudo-manifolds which can be stratified with only strata of even codimension, using the intersection homology theory. Furthermore they defined the Hirzebruch $L$-homology class. Our main purpose is to prove the intersection formula for Hirzebruch $L$-homology classes, which is the analogy of the Stiefel-Whitney homology classes' version [11]. By simple calculation, the case of manifolds can be reduced to the product formula for cohomology characteristic classes of bundles.

Let $X$ and $Y$ be compact oriented $P L$-pseudo-manifolds, possibly with boundary, which can be stratified with only strata of even codimension (cf. [7; $\S 5]$ ). If $X$ and $Y$ are properly $P L$-embedded in an oriented $P L$-manifold $M$, and if they are mutually transverse in $M$, then the intersection $X \cap Y$ is an orientable $P L$-pseudo-manifold which can be stratified with only strata of even codimension (cf. Proposition 2.3). Then we denote by $X \cdot Y$ the intersection $X \cap Y$ with the canonical orientation. Let $a$ and $b$ be in $H_{*}(M, \partial M ; Q)$. To state our main theorem, we define $a \cdot b$ by $a \cdot b=[M] \cap\left(([M] \cap)^{-1} a \cup([M] \cap)^{-1} b\right)$. Let $f: X \rightarrow M, g: Y \rightarrow M$ and $h: X \cdot Y \rightarrow M$ be the inclusions. Our main theorem is the following:

THEOREM. With the above, the following holds:

$$
f_{*} L_{*}(X) \cdot g_{*} L_{*}(Y)=h_{*} L_{*}(X \cdot Y) \cap l^{*}(M),
$$

where $l^{*}(M)$ is the L-cohomology class of $M$.

We recall the definition of the Hirzebruch $L$-homology classes due to Goresky and MacPherson [7]. Let $\Omega_{*}^{e v}$ be the oriented cobordism ring of compact oriented $P L$-pseudo-manifolds which can be stratified with only strata of even codimension (cf. $[7 ; \S 5])$. Let $X$ be a compact $n$-dimensional oriented $P L$-pseudo-manifold without boundary which can be stratified with only strata of even codimension. Denote by $\sigma(X)$ the signature of $X([7])$. Then $\sigma: \Omega_{*}^{e v}$ $\rightarrow Z$ is a ring homomorphism ([8]). We denote by $\left[X, S^{k}\right]$ the set of homotopy classes of maps from $X$ to the $k$-sphere $S^{k}$. Define a map $\theta:\left[X, S^{k}\right] \rightarrow \boldsymbol{Z}$ by

Received April 8, 1988, Revised July 20, 1988 
$\theta(f)=\sigma\left(f^{-1}(p)\right)$, where $f$ is transverse regular to $p$. Let $u$ be the generator of $H^{k}\left(S^{k}, \boldsymbol{Z}\right)$. For the case $2 k>n+1$, the Hirzebruch $L$-homology class $L_{k}(X)$ in $H_{k}(X, Q)$ is characterized by the following identity:

$$
\left\langle L_{k}(X), f^{*} u\right\rangle=\theta(f) \quad \text { for all } f \text { in }\left[X, S^{k}\right] \text {. }
$$

The restriction $2 k>n+1$ can be removed by crossing $X$ with a sphere, as in Milnor [13]. If $X$ has a boundary, we define $L_{*}(X)$ to be the pull back of $L_{*}(X \cup X)$, where $X \cup X$ is the double of $X$. If $X$ is a manifold, then $L_{*}(X)=$ $[X] \cap l^{*}(X)$ (cf. [13]).

\section{Transversality and classes of singularities.}

First we recall the definition of transversality according to Buoncristiano, Rourke and Sanderson [4].

Let $X$ be a polyhedron. Let $K$ be a collection of $P L$-balls in $X$. We write $|K|=\bigcup_{\sigma \in K} \sigma$. The collection $K$ is a ball complex structure ([4]) on $X$ if the following hold :

B1. $X$ is the disjoint union of the interiors Int $\sigma$ of all $P L$-balls $\sigma$ in $K$.

B2. If $\sigma$ is a $P L$-ball in $K$, then the boundary $\partial \sigma$ of $\sigma$ is the union of $P L$ balls in $K$.

Let $K$ be a ball complex structure on a $P L$-manifold $M$ and let $X$ be a subpolyhedron of $M$. We say that $X$ is collarable in $M$, if there exists a collar $c:(\partial M, X \cap \partial M) \times I \rightarrow(M, X)$. The polyhedron $X$ is transverse to $K$, if for each $P L$-ball $\sigma$ in $K$, the intersection $X \cap \sigma$ is collarable in $\sigma$. Let $X$ and $Y$ be subpolyhedra in $M$. We call the polyhedron $X$ transverse (or mock-transverse) to $Y$ in $M$, if there exists a ball complex structure $K$ on $M$ and exists a subcomplex $L$ of $K$ such that $|L|=Y$ and $X$ is transverse to $K([4])$. By McCrory [12], we know that for collarable polyhedra $X$ and $Y$ in an ambient $P L$-manifold, the polyhedron $X$ is transverse to $Y$ if and only if $Y$ is transverse to $X$. Other definitions of transversality were given by Armstrong [3], Stone [16] and McCrory [12]. These definitions are equivalent if subpolyhedra are collarable in an ambient $P L$-manifold (McCrory [12]).

Let $X$ be a subpolyhedron and $N$ be a $P L$-submanifold in a $P L$-manifold. The polyhedron $X$ is block transverse to $N$ if there exists a normal block bundle $\nu=(E, i, N)$ of $N$ such that the restriction $(X \cap E, i \mid(X \cap N), X \cap N)$ of $\nu$ to $X \cap N$ is a block bundle over $X \cap N$ (cf. [14]). Then by [4] we have the following :

Proposition 2.1. The polyhedron $X$ is block transverse to $N$ if and only if $N$ is transverse to $X$.

We need the following to prove our theorem (cf. [11]).

Lemma 2.2. Let $X$ and $Y$ be collarable subpolyhedra in a PL-manifold $M$ 
and $V$ a proper PL-submanifold in $M$. Suppose that $X$ is transverse to $Y$ and $V$ is transverse to $X \cup Y$ in $M$. Then $X \cap V$ is transverse to $Y \cap V$ in $V$.

LEMMA 2.3. Let $X$ and $Y$ be collarable subpolyhedra in a PL-manifold $M$ and $V$ be a proper PL-submanifold in $M$ with a normal block bundle $\nu=(E, i, V)$. Let $X$ be transverse to $Y$ and let $X \cup Y$ be block transverse to $\nu$. Then $X \cap V$ and $Y \cap V$ are transverse to $Y \cap E$ and $X \cap E$ in $E$, respectively.

Transversality Theorem 2.4 ([4], $[14])$. Let $X$ and $Y$ be collarable subpolyhedra of a PL-manifold $M$ and let $X \cap \partial M$ be transverse to $Y \cap \partial M$ in $\partial M$. Then there exists an arbitrarily small ambient isotopy $h_{t}$ of $M$ such that $h_{t} \mid \partial M$ is the identity for all $t$ and that $h_{1}(X)$ is transverse to $Y$ in $M$.

Next we recall the definition of classes of singularities due to [1] and [4]. Let $X$ and $Y$ be polyhedra. We denote by $X * Y$ the join of $X$ and $Y$. Let $\varsigma$ be a class of compact polyhedra. Let $c$ be a point. We define $c * \subseteq$ by $c * \widetilde{S}=$ $\{c * X \mid X \in \subseteq\}$. A class $\mathfrak{S}^{n}$ of polyhedra of pure dimension $n$ is called a class of singularities (cf. Akin [1], Buoncristiano, Rourke and Sanderson [4]) if the following hold:

S1. If $X \in \widetilde{S}^{n}$ and $Y=X$, then $Y \in \widetilde{S}^{n}$.

S2. $\phi \in \widetilde{S}^{-1}$.

S3. $X \in \mathbb{S}^{n}$ if and only if $S^{0} * X \in \mathfrak{S}^{n+1}$.

S4. If $X \in \mathfrak{S}^{m}$ and $Y \in \mathfrak{S}^{n}$, then $X * Y \in \mathfrak{S}^{m+n+1}$.

S5. $\mathfrak{S}^{m} \cap c * \mathbb{S}^{m-1}=\phi$.

Put $\subseteq=\left\{\varsigma^{n}\right\}$. We also call $\subseteq$ a class of singularities.

Let $\subseteq$ be a class of singularities. Let $X$ be a polyhedron of pure dimension $n$ and let $\partial X$ be a subpolyhedron. The polyhedron $X$ is called a S-space if the following hold:

1. $\partial X$ is of pure dimension $(n-1)$ or empty.

2. $\operatorname{Link}(x, X)$ is in $\mathbb{S}^{n-1}$ for $x$ in $X-\partial X$.

3. $\operatorname{Link}(x, X)$ is in $c * \mathbb{S}^{n-2}$ for $x$ in $\partial X$ if $\partial X$ is not empty.

4. $\operatorname{Link}(x, \partial X)$ is in $\mathfrak{S}^{n-2}$ for $x$ in $\partial X$ if $\partial X$ is not empty.

We say that a S-space $X$ is properly $P L$-embedded in a $P L$-manifold $M$ if $\partial M \cap X=\partial X$.

Proposition 2.5. Let $X$ and $Y$ be S-spaces properly PL-embedded in a PLmanifold $M$. If $X$ is transverse to $Y$ in $M$, then $X \cap Y$ is a S-space.

In order to prove this proposition, we will introduce some notations. Let $L$ be a ball complex. We assume that, for all $P L$-ball $\Delta$ in $L$, collars $c_{\Delta}: \partial \Delta$ $\times I \rightarrow \Delta$ are given. Put $\operatorname{Star}^{k}(\Delta)=\left\{\Delta^{\prime} \in L \mid \Delta^{\prime}>\Delta, \operatorname{dim} \Delta^{\prime}=\operatorname{dim} \Delta+k\right\}$ and put $\left|\operatorname{Star}^{k}(\Delta)\right|=\cup \Delta^{\prime}$, where $\Delta^{\prime}$ runs over all $P L$-balls in $\operatorname{Star}^{k}(\Delta)$. For a polyhedron $A$ in $\Delta$, we construct a subpolyhedron $P^{k}(A)$ in $\left|\operatorname{Star}^{k}(\Delta)\right|$ as follows :

First we put $P^{0}(A ; L)=A$. Next assume that $P^{k-1}(A ; L)$ is constructed. Then we put $P^{k}(A ; L)=\cup c_{\Delta^{\prime}}\left(\left(P^{k-1}(A) \cap \Delta^{\prime}\right) \times I\right)$, where $\Delta^{\prime}$ runs over all $P L$-balls 
in $\operatorname{Star}^{k}(\Delta)$. By the construction, we obtain the following:

Lemma 2.6. Let $c$ be a point in $A$. Then $P^{k}(A ; L)=P^{k}(c ; L) \times A$.

Proof of Proposition 2.5. Let $K$ be a ball complex structure on $M$ and let $L$ be a subcomplex of $K$ such that $|L|=Y$ and $X$ is transverse to $K$. First we prove that, for each $P L$-ball $\Delta$ in $K$, the intersection $X \cap \Delta$ is an empty set or a S-space with the boundary $X \cap \partial \Delta$, by induction on the codimension of $\Delta$ in $M$. If $\operatorname{dim} \Delta=\operatorname{dim} M$, it is clear. Assume that, for each $\Delta^{n-k}$ in $K$, the intersection $X \cap \Delta^{n-k}$ is a S-space with the boundary $X \cap \partial \Delta^{n-k}$. For any $\Delta^{n-k-1}$ in $K$, there exists an $(n-k)$-dimensional $P L$-ball $\Delta^{n-k}$ in $K$ such that $\Delta^{n-k}>$ $\Delta^{n-k-1}$. Since $X \cap \Delta^{n-k-1}=\left(X \cap \partial \Delta^{n-k}\right) \cap \Delta^{n-k-1}$, we can see that $X \cap \Delta^{n-k-1}$ is a ธ-space. Then $X \cap \Delta$ is a 5 -space for each $\Delta$ in $K$.

Let $K^{\prime}$ be a subdivision of $K$ which contains a triangulation of $X$. Let $L^{\prime}$ be a subcomplex of $K^{\prime}$ which is a subdivision of $L$. Let $\Delta$ be a $P L$-ball in $L$. Let $\tau$ be a simplex in $K^{\prime}\left|X \cap \Delta-K^{\prime}\right| X \cap \partial \Delta$. Let $c$ be a vertex of $\tau$ and let $\sigma$ be a simplex such that $\tau=c * \sigma$. Put $L_{c}=\operatorname{Link}\left(c ; K^{\prime} \mid X \cap \Delta\right)$. Then $P^{k}\left(c * L_{c} ; L\right)$ $=P^{k}(c ; L) \times c * L_{c}$ by Lemma 2.6 , where $k$ is the codimension of $\Delta$ in $L$. Let $\tilde{P}(c)$ and $\tilde{P}\left(c * L_{c}\right)$ be triangulations of $P^{k}(c ; L)$ and $P^{k}\left(c * L_{c} ; L\right)$, respectively. Since $X$ is transverse to $Y$, we have

Then

$$
\operatorname{Link}\left(\tau ; L^{\prime} \mid X\right)=\operatorname{Link}\left(\tau ; \tilde{P}\left(c * L_{c}\right)\right) .
$$

$$
\begin{aligned}
\operatorname{Link}\left(\tau ; L^{\prime} \mid X\right) & =\operatorname{Link}\left(c * \sigma ; \tilde{P}(c) \times c * L_{c}\right) \\
& =\operatorname{Link}(c ; \tilde{P}(c)) * \operatorname{Link}\left(\sigma ; c * L_{c}\right) .
\end{aligned}
$$

The fact that $X \cap \Delta$ is a $\subseteq$-space implies that $\operatorname{Link}\left(\sigma ; c * L_{c}\right)$ is an element in ऽ. On the other hand, $Y$ is a S-space. Then $\operatorname{Link}(\sigma ; \widetilde{P}(c))$ is an element in

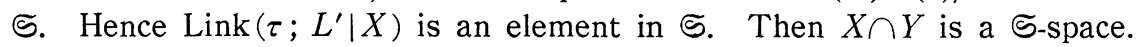

q.e.d.

Denote by $\mathcal{E}_{0}^{n}$ the class of compact oriented $n$-dimensional $P L$-pseudo-manifolds without boundary which can be stratified with only strata of even codimension. Put $\mathcal{E}_{0}=\left\{\mathcal{E}_{0}^{n}\right\}$. Define $\widetilde{S}\left(\mathcal{E}_{0}\right)$ by $\widetilde{S}^{n}\left(\mathcal{E}_{0}\right)=\cup\left\{S^{n-2 \imath} * X^{2 \imath-1} \mid X^{2 \imath-1} \in \mathcal{E}_{0}^{2 \imath-1}\right\}$. Then $\widetilde{S}\left(\mathcal{E}_{0}\right)$ is a class of singularities. Furthermore orientable $\mathfrak{S}\left(\mathcal{E}_{0}\right)$-spaces coincide with orientable $P L$-pseudo-manifolds which can be stratified with only strata of even codimension. Consequently, we can see the following from Proposition 2.5 .

Proposition 2.7. Let $X$ and $Y$ be compact orcented PL-pseudo-manifolds which can be stratified with only strata of even codimension. If $X$ and $Y$ are $P L$-embedded in an oriented PL-manifold $M$ and $X$ is transverse to $Y$ in $M$, then $X \cap Y$ is a compact oriented PL-pseudo-manifold which can be stratified with only strata of even codimension. 
Let $\subseteq$ be a class of singularities. Then the bordism theory of $\subseteq$-spaces is a $\boldsymbol{Z}_{2}$-homology theory (Akin [1]). If each of $\boldsymbol{S}_{\text {-spaces }}$ is an orientable $P L$ pseudo-manifold, then the oriented bordism theory $\Omega_{*}^{\Im}$ of $\mathfrak{S}_{\text {-spaces }}$ is a $\boldsymbol{Z}$ homology theory. We denote by $\Omega_{*}^{e v}$ the bordism theory of compact oriented $P L$-pseudo-manifolds which can be stratified with only strata of even codimension (Goresky and MacPherson [7], [8]). We need the following lemma, to prove Lemmas 4.1 and 4.7 .

LEMMA 2.8 ([5]). Let $h_{*}$ be $\Omega_{*}$ or $\Omega_{*}^{e v}$. For a pair $(A, B)$ of polyhedra, the following hold:

1. $\pi: h_{n}(A, B) \otimes Q \rightarrow H_{n}(A, B ; Q)$ is a surjection, where $\pi(\varphi, V)=\varphi_{*}[V]$.

2. There exists a natural transformation $T: h_{n}(A, B) \otimes Q \rightarrow \sum_{i=0}^{n} H_{n-i}(A, B$; $\left.h_{i} \otimes Q\right)$ and there exist bases $\left(\varphi_{\lambda}^{n-\imath} \circ p_{\lambda}, U_{\lambda}^{n-\imath} \times W_{\lambda}^{i}\right)$ of $h_{n}(A, B) \otimes Q$ such that $T\left(\varphi_{\lambda}^{n-\imath} \circ p_{\lambda}, U_{\lambda}^{n-\imath} \times W_{\lambda}^{i}\right)=\varphi_{\lambda}^{n-\imath} *\left[U_{\lambda}^{n-i}\right] \otimes W_{\lambda}^{i}$ and they are bases of $\sum_{i=0}^{n} H_{n-i}(A, B$; $\left.h_{\imath} \otimes Q\right)$.

Proof. First we prove the statement 2. Let $T_{1}: \pi_{n-i}^{s}(A, B) \otimes h_{i} \otimes Q \rightarrow$ $h_{n}(A, B) \otimes Q$ and $T_{2}: \pi_{n-i}^{s}(A, B) \otimes h_{i} \otimes Q \rightarrow H_{n-i}\left(A, B ; h_{i} \otimes Q\right)$ be natural transformations $([5 ; \S 1])$, where $\pi_{*}^{s}(A, B)$ is the stable homotopy group of $(A, B)$. Then $T_{1}: \sum_{l=0}^{n} \pi_{n-i}^{s}(A, B) \otimes h_{i} \otimes Q \rightarrow h_{n}(A, B) \otimes Q$ and $T_{2}: \sum_{\imath=0}^{n} \pi_{n-i}^{s}(A, B) \otimes h_{i} \otimes Q$ $\rightarrow \sum_{\imath=0}^{n} H_{n-i}\left(A, B ; h_{i} \otimes Q\right)$ are isomorphisms $\left(\left[5 ; \S 3\right.\right.$, Corollary 3]). Put $T=T_{2}$ 。 $T_{1}^{-1}$. Then $T: h_{n}(A, B) \otimes Q \rightarrow \sum_{i=0}^{n} H_{n-i}\left(A, B ; h_{i} \otimes Q\right)$ is the natural transformation. By the construction of $T$, we can obtain the bases which we want.

Next we show the statement 1 . Noting the construction of $T$, we can see that $\pi: h_{n}(A, B) \otimes Q \rightarrow H_{n}(A, B ; Q)$ coincides with $T: h_{n}(A, B) \otimes Q \rightarrow H_{n}(A, B$; $\left.h_{0} \otimes Q\right)$. Then $\pi$ is a surjection.

q.e.d.

We immediately have the following by the Künneth formula of ordinary homology and by Lemma 2.8. We need the following lemma to prove Lemma 4.3.

LeMma 2.9. Let $h_{*}$ be $\Omega_{*}$ or $\Omega_{*}^{e v}$. Let $(A, B)$ and $(C, D)$ be pairs of polyhedra. Then the cross product $\times: \sum_{i=0}^{n}\left(h_{n-i}(A, B) \times h_{i}(C, D)\right) \otimes Q \rightarrow h_{n}(A \times B$, $A \times D \cup C \times B) \otimes Q$ is a surjection, where $(\varphi, V) \times(\phi, U)=(\varphi \times \psi, V \times U)$.

\section{Axioms of Hirzebruch $L$-homology classes.}

Let $X$ and $Y$ be compact oriented $P L$-pseudo-manifolds which can be stratified with only strata of even codimension. Assume that $\operatorname{dim} X=\operatorname{dim} Y$. Let $f: X \rightarrow Y$ be an orientation preserving $P L$-embedding. We call $f$ a regular embedding if $f(X)$ is closed in $Y, f($ Int $X) \cap \partial Y=\phi$ and $f \mid \operatorname{Int} X$ is an open map, where Int $X=X-\partial X$.

Given a regular embedding $f: X \rightarrow Y$, we define a homomorphism $f^{\#}: H_{*}(Y, \partial Y ; Q) \rightarrow H_{*}(X, \partial X ; Q) \quad$ by $f^{\#}=\left(f_{*}\right)^{-1} \circ i_{*}, \quad$ where $\quad i:(Y, \partial Y) \rightarrow$ 
$(Y, Y-f(\operatorname{Int} X)) \quad$ is the inclusion. Note that $f_{*}: H_{*}(X, \partial X ; Q) \rightarrow$ $H_{*}(Y, Y-f(\operatorname{Int} X) ; Q)$ is an isomorphism by the excision property. Therefore $f^{\#}$ is well defined.

Let $\mathcal{E}$ be the category whose objects are compact oriented $P L$-pseudo-manifolds, possibly with boundary, which can be stratified with only strata of even codimension and whose morphisms are regular embeddings.

For each object $X$ in $\mathcal{E}$ with $\operatorname{dim} X=n$, we consider a (total) homology class

$$
L_{A}(X)=L_{0}(X)+L_{1}(X)+\cdots+L_{n}(X) \quad \text { in } \quad H_{*}(X, \partial X ; Q)
$$

satisfying the following axioms :

L0. $L_{n}(X)=[X]$.

L1. For every object $X$ of $\mathcal{E}$, the homology class $L_{\imath}(X)$ is in $H_{i}(X, \partial X ; Q)$ such that $L_{n-i}(X)=0$ if $i \not \equiv 0(\bmod 4)$.

L2. If $f: X \rightarrow Y$ is a morphism in $\mathcal{E}$, then $L_{A}(X)=f^{\#} L_{A}(Y)$.

L3. $L_{A}(X \times Y)=L_{A}(X) \times L_{A}(Y)$.

L4. If $\partial X=\phi$, then $\left\langle L_{A}(X), 1^{\circ}\right\rangle=\sigma(X)$, where $\sigma(X)$ is the signature of $X$.

We call such a homology class $L_{A}(X)$ an axiomatic $L$-homology class of $X$.

Theorem 3.1. Let $X$ be a compact oriented PL-pseudo-manifold which can be stratified with only strata of even codimension. Then the axiomatic $L$ homology class of $X$ concides with the Hirzebruch L-homology class of $X$.

We will prove the existence of axiomatic $L$-homology classes in Section 4. (cf. Lemma 4.3 and Corollaly 4.4).

LEMMA 3.2. If axiomatic L-homology classes exist, they coincide with the Hirzebruch L-homology class.

Proof. Considering Axiom L2, we may assume that $X$ has no boundary. Let $\theta:\left[X, S^{n}\right] \rightarrow \boldsymbol{Z}$ be the map which is used to define the Hirzebruch $L$ homology class in Section 1. Let $f: X \rightarrow S^{n}$ be an element of $\left[X, S^{n}\right]$. Then there exists a $P L$-embedding $f^{\prime}: X \rightarrow S^{n} \times D^{k}$ such that $f^{\prime} \simeq f \times\{0\}$ for $k$ sufficiently large. Let $p t \times \iota: D^{k} \rightarrow S^{n} \times D^{k}$ be the embedding defined by $(p t \times \iota)(x)=$ $(p t, x)$, where $p t$ is a point of $S^{n}$. Let $\nu=\left(E, i, D^{k}\right)$ be a normal bundle of $p t \times \iota$. On the other hand, we can assume that $f^{\prime}(X)$ is transverse to $\nu$. For simplicity, we put

and

$$
X \cap D^{k}=f^{\prime}(X) \cap(p t \times \iota)\left(D^{k}\right),
$$

$$
X \cdot D^{k}=f^{\prime}(X) \cdot\left(p t \times_{\iota}\right)\left(D^{k}\right) .
$$

Since $\nu$ is a trivial bundle, we have the following commutative diagram: 


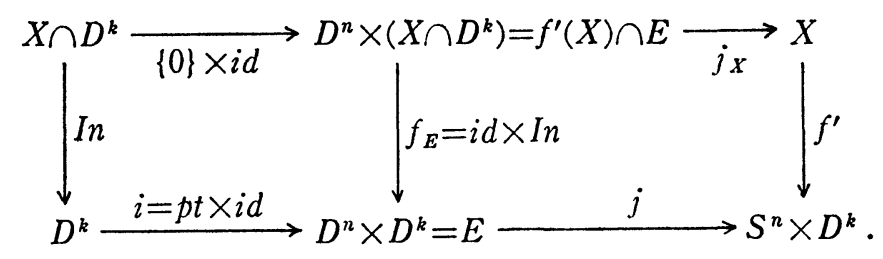

Here $j, f_{E}$ and $I n$ are the inclusions and $j_{X}$ is defined by $j_{X}(x)=f^{\prime-1}(x)$ for $x$ in $f^{\prime}(X) \cap E$. Let $u$ be the generator of $H^{n}\left(S^{n} ; \boldsymbol{Z}\right)$. Assume that $\operatorname{dim} X=m$ and put $\varepsilon=(-1)^{(n+k-m) \cdot n}$. Then

$$
\begin{aligned}
\left\langle L_{A}(X), f^{*} u\right\rangle & =\left\langle f_{*}^{\prime} L_{A}(X),\left(\left[S^{n} \times D^{k}\right] \cap\right)^{-1} \jmath_{*} i_{*}\left[D^{k}\right]\right\rangle \\
& =\varepsilon\left\langle j_{*} i_{*}\left[D^{k}\right],\left(\left[S^{n} \times D^{k}\right] \cap\right)^{-1} f_{*}^{\prime} L_{A}(X)\right\rangle \\
& =\varepsilon\left\langle i_{*}\left[D^{k}\right], j^{*}\left(\left[S^{n} \times D^{k}\right] \cap\right)^{-1} f_{*}^{\prime} L_{A}(X)\right\rangle .
\end{aligned}
$$

Note that $j^{*}\left(\left[S^{n} \times D^{k}\right] \cap\right)^{-1} f_{*}^{\prime}=([E] \cap)^{-1} f_{E *} j_{X}^{\#}$. By Axiom L2, we have $j_{X}^{\#} L_{A}(X)$ $=L_{A}\left(f^{\prime}(X) \cap E\right)$. Then

$$
\left\langle L_{A}(X), f^{*} u\right\rangle=\varepsilon\left\langle i_{*}\left[D^{k}\right],([E] \cap)^{-1} f_{E *} L_{A}\left(f^{\prime}(X) \cap E\right)\right\rangle .
$$

We note that $f^{\prime}(X) \cap E=D^{n} \times\left(X \cdot D^{k}\right)$. By Axioms L0 and L3, we have $L_{A}\left(f^{\prime}(X) \cap E\right)=L_{A}\left(D^{n} \times\left(X \cdot D^{k}\right)\right)=L_{A}\left(D^{n}\right) \times L_{A}\left(X \cdot D^{k}\right)=\left[D^{n}\right] \times L_{A}\left(X \cdot D^{k}\right)$. Then

Thus

$$
\begin{aligned}
f_{E *} L_{A}\left(f^{\prime}(X) \cap E\right) & =(i d \times I n)_{*}\left(\left[D^{n}\right] \times L_{A}\left(X \cdot D^{k}\right)\right) \\
& =\left[D^{n}\right] \times I n_{*} L_{A}\left(X \cdot D^{k}\right) .
\end{aligned}
$$

$$
\begin{aligned}
\left\langle L_{A}(X), f^{*} u\right\rangle & \left.=\left\langle\left[D^{n}\right] \times \operatorname{In}_{*} L_{A}\left(X \cdot D^{k}\right),([E] \cap)^{-1} i_{*}\left[D^{k}\right]\right)\right\rangle \\
& =\left\langle\operatorname{In}_{*} L_{A}\left(X \cdot D^{k}\right), 1^{0}\right\rangle=\left\langle L_{A}\left(X \cdot D^{k}\right), 1^{0}\right\rangle .
\end{aligned}
$$

By Axiom L4, we have $\left\langle L_{A}(X), f^{*} u\right\rangle=\sigma\left(X \cdot D^{k}\right)$. Since $\theta(f)=\sigma\left(X \cdot D^{k}\right)$, we have $\left\langle L_{A}(X), f^{*} u\right\rangle=\theta(f)$. Thus $L_{A}(X)$ is the Hirzebruch $L$-homology class.

q.e.d.

\section{Characterization of Hirzebruch $L$-homology classes.}

Let $X$ be a compact oriented $P L$-pseudo-manifold which can be stratified with only strata of even codimension. Let $M$ be an oriented $P L$-manifold. Let $\tilde{M}$ and $\bar{M}$ be codimension zero submanifolds of $\partial M$ such that $\partial M=\tilde{M} \cup \bar{M}$ and $\tilde{M} \cap \bar{M}=\partial \tilde{M}=\partial \bar{M}$. Let $\Omega_{*}$ be the oriented bordism theory of compact differentiable manifolds. Let $\Omega_{*}^{e v}$ be the oriented bordism theory of compact oriented $P L$-pseudo-manifolds which can be stratified with only strata of even codimension. Throughout this section, we use the above notation.

Let $X$ be $P L$-embedded in $M$ such that $\partial X \subset \tilde{M}$ and $X-\partial X \subset M-\partial M$. Let $f:(X, \partial X) \rightarrow(M, \tilde{M})$ be the inclusion. We define homomorphisms 


$$
\sigma_{f}: \Omega_{*}(M, \bar{M}) \otimes Q \longrightarrow Q, \quad \text { and } \quad \bar{\sigma}_{f}: \Omega_{*}^{e v}(M, \bar{M}) \otimes Q \longrightarrow Q .
$$

Let $b: \Omega_{*}(M, \bar{M}) \rightarrow \Omega_{*}^{e v}(M, \bar{M})$ be the natural map. If $\bar{\sigma}_{f}$ is defined, we define $\sigma_{f}$ by $\sigma_{f}=\bar{\sigma}_{f} \circ b$. Let $\varphi: V \rightarrow M$ be a map in $\Omega_{*}^{e v}(M, \bar{M})$. Then there exists a $P L$-embedding $\phi:(V, \partial V) \rightarrow\left(M \times D^{\alpha}, \bar{M} \times D^{\alpha}\right)$, for $\alpha$ sufficiently large, such that $\phi \simeq \varphi \times\{0\}$. By using the transversality theorem, we can assume that $\phi(V)$ is transverse to $X \times D^{\alpha}$ in $M \times D^{\alpha}$. By Proposition 2.7 the intersection $\phi(V) \cap$ $\left(X \times D^{\alpha}\right)$ is an oriented $P L$-pseudo-manifold which can be stratified with only strata of even codimension. We denote by $\psi(V) \cdot\left(X \times D^{\alpha}\right)$ the intersection $\psi(V) \cap\left(X \times D^{\alpha}\right)$ with the canonical orientation. Therefore we define $\bar{\sigma}_{f}(\varphi, V)$ to be the signature $\sigma\left(\psi(V) \cdot\left(X \times D^{k}\right)\right)$. By the transversality theorem and Proposition 2.7, we can well define $\bar{\sigma}_{f}$. We assume that $\operatorname{dim} X=n$ and $\operatorname{dim} M$ $=n+k$.

LEMMA 4.1. With the above situation, there exists a unique cohomology class $\Phi(f)=\Phi^{0}+\Phi^{1}+\cdots+\Phi^{n+k}$ in $H^{*}(M, \bar{M}: Q)$ satısfying the following:

$$
\left\langle\varphi_{*}\left([V] \cap l^{*}(V)\right), \Phi(f)\right\rangle=\sigma_{f}(\varphi, V) \quad \text { for each }(\varphi, V)
$$

in $\Omega_{*}(M, \bar{M}) \otimes Q$. Here $l^{*}(V)$ is the Hirzebruch L-cohomology class of $V$. Furthermore $\Phi^{k+\imath}=0$ if $i \neq 0(\bmod 4)$ or $i<0$.

Proof. We will inductively define cohomology classes $\Phi^{\imath}$ in $H^{\imath}(M, \bar{M} ; Q)$ for $\imath=0,1, \cdots, n+k$, where $\operatorname{dim} X=n$ and $\operatorname{dim} M=n+k$. Note that we can choose bases of $\Omega_{*}(M, \bar{M}) \otimes Q$ in $\Omega_{*}(M, \bar{M})$. First we define $\widetilde{\Phi}^{0}: \Omega_{0}(M, \bar{M}) \otimes Q$ $\rightarrow Q$ by $\tilde{\Phi}^{0}\left(\varphi, V^{0}\right)=\sigma_{f}\left(\varphi, V^{0}\right)$ for $\left(\varphi, V^{0}\right)$ in $\Omega_{0}(M, \bar{M})$. Let us define $p_{\imath}: \Omega_{\imath}(M, \bar{M}) \otimes Q \rightarrow H_{i}(M, \bar{M} ; Q)$ by $p_{i}(\varphi, V)=\varphi_{*}[V]$. Then $p_{\imath}$ is a surjection by Lemma 2.8. Since $\varphi_{*}\left[V^{0}\right]=0$ implies $\tilde{\Phi}^{0}\left(\varphi, V^{0}\right)=0$, we see that $\tilde{\Phi}^{0}$ determines a cohomology class $\Phi^{0}$ in $H^{0}(M, \bar{M} ; Q)$ such that $\left\langle\varphi_{*}\left(\left[V^{0}\right] \cap l^{*}\left(V^{0}\right)\right), \Phi^{0}\right\rangle$ $=\sigma_{f}\left(\varphi, V^{0}\right)$. Next we assume that there exist cohomology classes $\Phi^{2}$ in $H^{\imath}(M, \bar{M} ; Q)$ for $i=0,1, \cdots, s-1$, such that $\left\langle\varphi_{*}\left(\left[V^{j}\right] \cap l^{*}\left(V^{j}\right)\right), \sum_{t=0}^{j} \Phi^{t}\right\rangle=$ $\sigma_{f}\left(\varphi, V^{j}\right)$ for each $\left(\varphi, V^{\jmath}\right)$ in $\Omega_{\jmath}(M, \bar{M})$ with $\jmath \leqq_{\imath}<s$. Define $\tilde{\Phi}^{s}: \Omega_{s}(M, \bar{M}) \otimes Q$ $\rightarrow Q$ by $\tilde{\Phi}^{s}\left(\varphi, V^{s}\right)=\sigma_{f}\left(\varphi, V^{s}\right)-\left\langle\varphi_{*}\left(\left[V^{s}\right] \cap l^{*}\left(V^{s}\right)\right), \sum_{t=0}^{s-1} \Phi^{t}\right\rangle$. We will prove that $\tilde{\Phi}^{s}$ determines a cohomology class. We assume that $\varphi_{*}\left[V^{s}\right]=0$. By Lemma 2.8 , there exist $\left(\varphi_{i}, U^{s-i}\right)$ in $\Omega_{s-i}(M, \bar{M}), W^{i}$ in $\Omega_{i}(p t)$ and $\alpha_{\imath}$ in $Q$ such that

$$
\left(\varphi, V^{s}\right)=\sum_{\imath=1}^{s} \alpha_{\imath}\left(\varphi_{i} \circ q, U^{s-\imath} \times W^{i}\right) \quad \text { in } \quad \Omega_{*}(M, \bar{M}) \otimes Q
$$

where $q: U^{s-\imath} \times W^{i} \rightarrow U^{s-\imath}$ is the projection. On the other hand, $\tilde{\Phi}^{s}\left(\varphi_{i} \circ q, U^{s-\imath} \times W^{i}\right)$ $=\sigma_{f}\left(\varphi_{i} \circ q, U^{s-\imath} \times W^{i}\right)-\left\langle\left(\varphi_{i} \circ q\right)_{*}\left(\left[U^{s-\imath} \times W^{i}\right] \cap l^{*}\left(U^{s-\imath} \times W^{i}\right)\right), \sum_{t=0}^{s-1} \Phi^{t}\right\rangle=\sigma\left(W^{i}\right)\left\{\sigma_{f}\left(\varphi_{i}\right.\right.$, $\left.\left.U^{s-\imath}\right)-\left\langle\varphi_{i *}\left(\left[U^{s-i}\right] \cap l^{*}\left(U^{s-i}\right)\right), \sum_{t=0}^{s-1} \Phi^{t}\right\rangle\right\}=0$. Then $\varphi_{*}\left[V^{s}\right]=0$ implies $\tilde{\Phi}^{s}\left(\varphi, V^{s}\right)$ $=0$. Note that $p_{s}: \Omega_{s}(M, \bar{M}) \times Q \rightarrow H_{s}(M, \bar{M} ; Q)$ is a surjection. Then $\tilde{\Phi}^{s}$ determines a cohomology class $\Phi^{s}$ in $H^{s}(M, \bar{M} ; Q)$ such that $\left\langle\varphi_{*}\left(\left[V^{s}\right] \cap l^{*}\left(V^{s}\right)\right)\right.$, $\left.\sum_{t=0}^{s} \Phi^{t}\right\rangle=\sigma_{f}\left(\varphi, V^{s}\right)$. Put $\Phi(f)=\Phi^{0}+\Phi^{1}+\cdots+\Phi^{n+k}$. By the construction of $\Phi(f)$, we have $\Phi^{k+\imath}=0$ if $\imath<0$ or $i \neq 0(\bmod 4)$.

q. e. d. 
Definition 4.2. Choose a $P L$-embedding of $X$ in $D^{N}$ for $N$ sufficiently large such that $X \cap \partial D^{N}=\partial X$. Let $M$ and $\tilde{M}$ be regular neighborhoods of $X$ and $\partial X$ in $D^{N}$ and $\partial D^{N}$, respectively. Let $f:(X, \partial X) \rightarrow(M, \tilde{M})$ be the inclusion. We define a homology class $L(X)$ in $H_{*}(X, \partial X ; Q)$ by $L(X)=f_{*}^{-1}([M] \cap \Phi(f))$, where $\Phi(f)$ is the cohomology class in Lemma 4.1. The cohomology class $L(X)$ does not depend on the choice of the embedding in $D^{N}$.

LEMma 4.3. Assume that $L(X)$ is the homology class as above. Then $L(X)$ satisfies Axioms L0, L1, L2, L3 and L4.

Lemmas 3.2 and 4.3 imply the following corollary, which includes Theorem 3.1 .

COROLlARY 4.4. The homology class $L(X)$, the axiomatic L-homology class and the Hirzebruch L-homology class coincide with each other.

Proof of Lemma 4.3. We can easily prove that $L(X)$ satisfies Axioms L0, L1 and L2. So we omit the proof. First we prove that $L(X)$ satisfies Axiom L4. For the case where $(\varphi, V)=(i d, M)$, we have $\sigma_{f}(i d, M)=\sigma(X)$. Since $M$ is a codimension zero submanifold of $D^{N}$, we have $[M] \cap l^{*}(M)=[M]$. Then $\sigma(X)=\left\langle[M],([M] \cap)^{-1} f_{*} L(X)\right\rangle=\left\langle f_{*} L(X), 1_{M}^{0}\right\rangle=\left\langle L(X), 1^{0}\right\rangle$.

Next we prove that $L(X)$ satisfies Axiom L3. Let $M_{X}$ and $M_{Y}$ be regular neighborhoods of $X$ and $Y$ in $D^{m+p}$ and $D^{n+q}$, respectively. Let $f_{X}:(X, \partial X) \rightarrow$ $\left(M_{X}, \partial M_{X}\right)$ and $f_{Y}:(Y, \partial Y) \rightarrow\left(M_{Y}, \partial M_{Y}\right)$ be the inclusions. We use the same notation as in the definition of $L(X)$. By calculation, we have $\left\langle(\varphi \times \phi)_{*}([V \times U]\right.$ $\left.\cap l^{*}(V \times U),\left(\left[M_{X} \times M_{Y}\right] \cap\right)^{-1}\left(f_{X} \times f_{Y}\right)_{*}(L(X) \times L(Y))\right\rangle=\left\langle\varphi_{*}\left([V] \cap l^{*}(V)\right)\right.$, $\left.\left(\left[M_{X}\right] \cap\right)^{-1} f_{X *} L(X)\right\rangle \times\left\langle\psi_{*}\left([V] \cap l^{*}(U)\right),\left(\left[M_{Y}\right] \cap\right)^{-1} f_{Y *} L(Y)\right\rangle=\sigma_{f_{X}}(\varphi, V) \cdot \sigma_{f_{Y}}(\psi, U)$ $=\sigma_{f_{X} \times f_{Y}}(\varphi \times \psi, V \times U)$. By Lemmas 2.9 and 4.1, we have $\Phi\left(f_{X} \times f_{Y}\right)=$ $\left(\left[M_{X} \times M_{Y}\right] \cap\right)^{-1}\left(f_{X} \times f_{Y}\right)_{*}(L(X) \times L(Y))$. By considering the definition of $L(X \times Y)$ (cf. Definition 4.2), we have $L(X) \times L(Y)=L(X \times Y)$.

q.e.d.

THEOREM 4.5. Let $X$ be PL-embedded in $M$ such that $\partial X \subset \tilde{M}, X \cap \partial M=\partial X$ and $X$ is collarable in $M$. Let $f:(X, \partial X) \rightarrow(M, \tilde{M})$ be the inclusion. Then, for each map $\varphi: V \rightarrow M$ in $\Omega_{*}(M, \bar{M})$, the following holds:

$$
\left\langle\varphi_{*}\left([V] \cap l^{*}(V)\right),([M] \cap)^{-1}\left(f_{*} L_{*}(X) \cap \bar{l}(M)\right)\right\rangle=\sigma_{f}(\varphi, V) .
$$

Furthermore the homology class $f_{*} L_{*}(X)$ is completely characterized by this identity. Here $i(M)$ is the inverse of $l^{*}(M)$, that is, $i(M) \cup l^{*}(M)=1$.

This theorem gives the fundamental characterization of Hirzebruch $L$ homology classes. We need both this theorem and the following proposition to prove our main theorem.

Proposition 4.6. With the same situation as in Theoren 4.5, the following holds: 


$$
\begin{aligned}
& \left\langle\varphi_{*} L_{*}(V),([M] \cap)^{-1}\left(f_{*} L_{*}(X) \cap \bar{l}(M)\right)\right\rangle=\bar{\sigma}_{f}(\varphi, V) \\
& \qquad \text { for each }(\varphi, V) \text { in } \Omega_{*}^{e v}(M, \bar{M}) .
\end{aligned}
$$

We need the following lemma to prove this proposition. For the proof of this lemma, we may replace $[V] \cap l^{*}(V), \sigma_{f}$ and $\Omega_{*}$ in Lemma 4.1 by $L_{*}(V)$, $\bar{\sigma}_{f}$ and $\Omega_{*}^{e v}$. Then we can apply the proof of Lemma 4.1 to that of the following lemma, using Corollary 4.3. So we omit the proof.

LEMMA 4.7. With the same situation as in Lemma 4.1, there exists a unique cohomology class $\Phi(f)=\Phi^{0}+\Phi^{0}+\cdots+\Phi^{n+k}$ in $H^{*}(M, \bar{M} ; Q)$ satisfying the following:

$$
\begin{aligned}
\left\langle\varphi_{*} L_{*}(V), \Phi(f)\right\rangle & =\bar{\sigma}_{f}(\varphi, V) \\
& \text { for each }(\varphi, V) \text { in } \Omega_{*}^{e v}(M, \bar{M}) .
\end{aligned}
$$

Furthermore $\Phi(f)$ coincides with that in Lemma 4.1.

Proof of Proposition 4.6. If $(\varphi, V)$ is in $\Omega_{*}(M, \bar{M})$, then $L_{*}(V)=[V] \cap l^{*}(V)$. Hence the cohomology class $\Phi(f)$ in Lemma 4.7 satisfies the identity in Lemma 4.1. By Theorem 4.5 and the uniqueness of $\Phi(f)$ in Lemma 4.1, we have $\Phi(f)$ $=([M] \cap)^{-1}\left(f_{*} L_{*}(X) \cap \bar{l}(M)\right)$.

q.e.d.

The following in this section is devoted to prove Theorem 4.5. To prove Theorem 4.5, we need to give a characterization of the dual Hirzebruch $L$ cohomology class $\bar{l}(\xi)$ of an oriented block bundle $\xi$.

Let $\xi=(E, \iota, B)$ be an oriented block bundle over a compact polyhedron $B$. Denote by $\bar{E}$ the total space of the sphere bundle associated with $\xi$. Let $U_{\xi}$ be the Thom class of $\xi$. We will define homomorphisms $\sigma_{\xi}: \Omega_{*}(E, \bar{E}) \otimes Q \rightarrow Q$ and $\bar{\sigma}_{\xi}: \Omega_{*}^{e v}(E, \bar{E}) \otimes Q \rightarrow Q$ as follows. We assume that $B$ is $P L$-embedded in $\boldsymbol{R}^{N}$. Let $A$ be a regular neighborhood of $B$ in $\boldsymbol{R}^{N}$. Denote by $p: A \rightarrow B$ the deformation retraction. Denote by $p^{*} \xi=\left(E\left(p^{*} \xi\right), \iota^{\prime}, A\right)$ the induced bundle. Let $(\bar{p}, p):\left(E\left(p^{*} \xi\right), A\right) \rightarrow(E, B)$ and $(\bar{i}, i):(E, B) \rightarrow\left(E\left(p^{*} \xi\right), A\right)$ be bundle maps, where $i$ and $i$ are the inclusions. Define $\sigma_{\xi}$ and $\bar{\sigma}_{\xi}$ by $\sigma_{\xi}(\varphi, V)=\sigma_{\iota^{\prime}}\left(i_{\circ} \varphi, V\right)$ and $\bar{\sigma}_{\xi}(\varphi, V)=\bar{\sigma}_{\iota^{\prime}}(i \circ \varphi, V)$.

Proposition 4.8. With the situation as above, the following holds:

$$
\left\langle\varphi_{*}\left([V] \cap l^{*}(V)\right), U_{\xi} \cup \iota^{*-1} \bar{l}(\xi)\right\rangle=\sigma_{\xi}(\varphi, V)
$$

for $(\varphi, V)$ in $\Omega_{*}(E, \bar{E})$. Furthermore the dual Hirzebruch L-honology class $\bar{l}(\xi)$ is completely characterized by this identity.

This proposition is the fundamental characterization of the dual Hirzebruch $L$-cohomology classes of bundles. We need this proposition only to prove the following proposition, which is necessary to prove Theorem 4.5 and our main theorem. 
Proposition 4.9. With the same situation as in Proposition 4.8, the follouing holds:

$$
\begin{aligned}
\left\langle\varphi_{*} L_{*}(V), U_{\xi} \cap c^{*-1} \bar{l}(\xi)\right\rangle= & \bar{\sigma}_{\hat{\xi}}(\varphi, V) \\
& \text { for }(\varphi, V) \text { in } \Omega_{*}^{e v}(E, \bar{E}) .
\end{aligned}
$$

Furthermore the dual Hirzebruch L-homology class $\bar{l}(\xi)$ is completely characterized by this identity.

Proof of Proposition 4.8. We use the notations which are used to define $\sigma_{\xi}$. Let $\varphi: V \rightarrow E$ be a map in $\Omega_{*}(E, \bar{E})$. Then there exists a $P L$-embedding $\phi: V \rightarrow E\left(p^{*} \xi\right)$ in $\Omega_{*}\left(E\left(p^{*} \xi\right), \bar{E}\left(p^{*} \xi\right)\right)$ such that $\psi \simeq i \circ \varphi$ and $\phi(V)$ is transverse to A. Since $\varphi=\bar{p} \circ \bar{\imath} \circ \varphi \simeq \bar{p} \circ \phi$, we have

$$
\begin{aligned}
&\left\langle\varphi_{*}\left([V] \cap l^{*}(V)\right), U_{\xi} \cup \iota^{*-1} \bar{l}(\xi)\right\rangle \\
&=\left\langle\phi_{*}\left([V] \cap l^{*}(V)\right), \bar{p}^{*} U_{\xi} \cup \bar{p}^{*} \iota^{*-1} \bar{l}(\xi)\right\rangle \\
&=\left\langle[V], \phi^{*} U_{p * \xi} \cap l^{*}(V) \cup \phi^{*} \iota^{*-1} \bar{l}\left(p^{*} \xi\right)\right\rangle \\
&=\left\langle[V] \cap \phi^{*} U_{p * \xi}, l^{*}(V) \cup \psi^{*} \iota^{*-1} \bar{l}\left(p^{*} \xi\right)\right\rangle .
\end{aligned}
$$

Let $j: \phi(V) \cap A \rightarrow V$ be defined by $j(x)=\phi^{-1}(x)$. Then $[V] \cap \phi^{*} U_{p^{*} \xi}=j_{*}[\psi(V) \cdot A]$. On the other hand, we have $j^{*} l^{*}(V) \cup j^{*} \psi^{*} \iota^{*-1} \bar{l}\left(p^{*} \xi\right)=l^{*}(\phi(V) \cdot A)$. Then $\left\langle\varphi_{*}\left([V] \cap l^{*}(V)\right), U_{\xi} \cup \iota^{*-1} \bar{l}(\xi)\right\rangle=\left\langle[\psi(V) \cdot A], l^{*}(\phi(V) \cdot A)\right\rangle$. Noting that $\phi(V) \cdot A$ is an oriented $P L$-manifold, we have $\left\langle[\psi(V) \cdot A], l^{*}(\psi(V) \cdot A)\right\rangle=\sigma(\psi(V) \cdot A)$. Consequently $\left\langle\varphi_{*}\left([V] \cap l^{*}(V)\right), U_{\xi} \cup \iota^{*-1} \bar{l}(\xi)\right\rangle=\sigma_{\iota^{\prime}}(\bar{\imath} \circ \varphi)=\sigma_{\xi}(\varphi, V)$ for each $(\varphi, V)$ in $\Omega_{*}(E, \bar{E})$.

Replacing $f$ by $\iota^{\prime}$, we can see that the uniqueness of $\Phi(f)$ in Lemma 4.1 implies the uniqueness of $\bar{l}(\xi)$.

q.e.d.

Proof of Proposition 4.9. By Lemma 4.7, there exists a unique cohomology class $\Phi(i \circ \varphi)$ such that $\left\langle\varphi_{*} L_{*}(V), \Phi(\bar{\imath} \circ \varphi)\right\rangle=\bar{\sigma}_{\iota^{\prime}}(\bar{i} \circ \varphi, V)=\bar{\sigma}_{\xi}(\varphi, V)$ for each $(\varphi, V)$ in $\Omega_{*}^{e v}(E, \bar{E})$. If $V$ is an oriented $P L$-manifold, then $L_{*}(V)=[V] \cap l^{*}(V)$ and $\bar{\sigma}_{\xi}(\varphi, V)=\sigma_{\xi}(\varphi, V)$. By using Proposition 4.8 , we have

$$
\Phi(\bar{\imath} \circ \varphi)=U_{\xi} \cup \iota^{*-1} \bar{l}(\xi) .
$$

q.e.d.

Proof of Theorem 4.5. Let $(\varphi, V)$ be a map in $\Omega_{*}(M, \bar{M})$. Let $\phi:(V, \partial V)$ $\rightarrow\left(M \times D^{k}, \bar{M} \times D^{k}\right)$ be a $P L$-embedding for $k$ sufficiently large, such tha $\phi \simeq \varphi \times\{0\}$ and $\phi(V)$ is transverse to $(f \times \imath d)\left(X \times D^{k}\right)$. Then

$$
\begin{aligned}
& \left\langle\varphi_{*}\left([V] \cap l^{*}(V)\right),([M] \cap)^{-1}\left\{f_{*} L_{*}(X) \cap \bar{l}(M)\right\}\right\rangle \\
& =\left\langle\phi_{*}\left([V] \cap l^{*}(V)\right),\left(\left[M \times D^{k}\right] \cap\right)^{-1}\left\{(f \times i d)_{*} L_{*}\left(X \times D^{k}\right) \cap \bar{l}\left(M \times D^{k}\right)\right\}\right\rangle .
\end{aligned}
$$

Therefore we may prove the case where $\varphi$ is a $P L$-embedding and $\varphi(V)$ is transverse to $f(X)$.

We assume that $\varphi: V \rightarrow M$ is a $P L$-embedding with a normal block bundle 
$\nu=\left(E, \varphi_{E}, V\right)$ and that $X$ is transverse to $\nu$. Let $U_{\nu}$ be the Thom class of $\nu$, that is $[E] \cap U_{\nu}=\varphi_{E *}[V]$. Let $\bar{E}$ be the total space of the sphere bundle associated with $\nu$. Put $\hat{X}=c l(X-E)$ and $\hat{M}=c l(M-E)$. Let $j:(M, \tilde{M}) \rightarrow(M, \hat{M})$, $j_{E}:(E ; \bar{E}, \tilde{E}) \rightarrow(M ; \hat{M}, \bar{M}), i:(X \cap E, \partial(X \cap E)) \rightarrow(X, \hat{X}), j_{X}:(X, \partial X) \rightarrow(X, \hat{X})$, $f_{E}:(X \cap E, \partial(X \cap E)) \rightarrow(E, \bar{E})$ and $\hat{f}:(X, \hat{X}) \rightarrow(M, \hat{M})$ be the inclusions. Then we have the following commutative diagram:

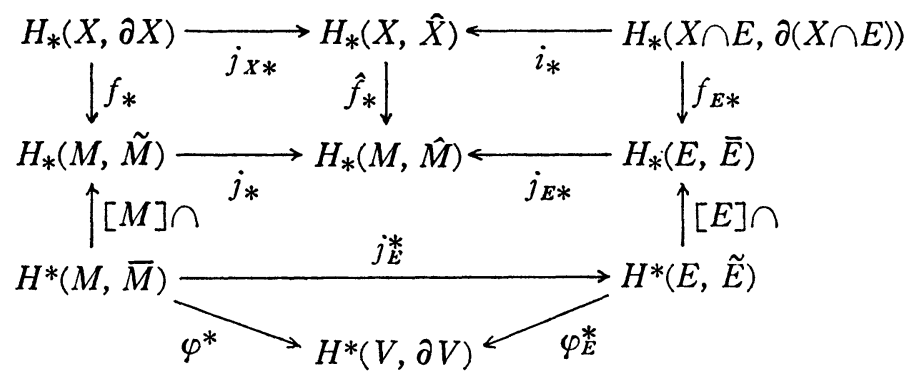

Put $P=\left\langle\varphi_{*}\left([V] \cap l^{*}(V)\right),([M] \cap)^{-1}\left\{f_{*} L_{*}(X) \cap \bar{l}(M)\right\}\right\rangle$. Then $P=\langle[V]$, $\left.l^{*}(V) \cup \varphi^{*}([M] \cap)^{-1}\left\{f_{*} L_{*}(X) \cap \bar{l}(M)\right\}\right\rangle$. Note that $[E] \cap U_{\nu}=\varphi_{E *}[V]$. Put $\varepsilon=(-1)^{\operatorname{codim} V \cdot \operatorname{codim} X}$. Then $P=\varepsilon\left\langle[E] \cap \varphi_{E^{*-1}}^{*} \varphi^{*}([M] \cap)^{-1}\left\{f_{*} L_{*}(X) \cap \bar{l}(M)\right\}\right.$, $\left.U_{\nu} \cup \varphi_{E}^{*-1} l^{*}(V)\right\rangle$. By the above commutative diagram, we have $([E] \cap) \varphi_{E}^{*-1} \varphi^{*}$. $\left.([M] \cap)^{-1}=\jmath_{E *}^{-1}\right]_{*}$. Then

$$
\begin{aligned}
P & =\varepsilon\left\langle j_{E *}^{-1} j_{*}\left(f_{*} L_{*}(X) \cap i(M)\right), U_{\nu} \cup \varphi_{E}^{*-1} l^{*}(V)\right\rangle \\
& =\varepsilon\left\langle j_{E}^{-1} j_{*} f_{*} L_{*}(X) \cap j_{E}^{*} \bar{l}(M), U_{\nu} \cup \varphi_{E}^{*-1} l^{*}(V)\right\rangle \\
& =\varepsilon\left\langle j_{E *}^{-1} j_{*} f_{*} L_{*}(X), U_{\nu} \cup j_{E}^{*} \bar{l}(M) \cup \varphi_{E}^{*-1} l^{*}(V)\right\rangle .
\end{aligned}
$$

By the above commutative diagram, we have $j_{E *}^{-1} j_{*} f_{*}=f_{E *{ }_{*}^{*}}^{-1} j_{X *}$. Then we have $i_{*}^{-1} j_{X *} L_{*}(X)=i^{\#} L_{*}(X)=L_{*}(X \cap E)$ by Axiom L2. On the other hand, we have $j_{E}^{*} \bar{l}(M) \cup \varphi_{E}^{*-1} l^{*}(V)=\bar{l}(E) \cup \varphi_{E}^{*-1} l^{*}(V)=\varphi_{E}^{*-1} l(V)$. By the above, we have $P=\varepsilon\left\langle f_{E *} L_{*}(X \cap E), U_{\nu} \cup \varphi_{E}^{*-1} \bar{l}(\nu)\right\rangle$. By Proposition 4.9, we have $P=\varepsilon \bar{\sigma}_{\nu}\left(f_{E}, X \cap E\right)$. In view of the definitions of $\bar{\sigma}_{\nu}$ and $\sigma_{f}$, we have $P=\varepsilon \sigma((X \cap E) \cdot V)=\sigma(V \cdot X)=$ $\sigma_{f}(\varphi, V)$. Furthermore by Lemma 4.1 , we can see the uniqueness of $f_{*} L_{*}(X)$.

q.e.d.

\section{Proof of Theorem.}

In order to prove our theorem, we need the following Halperin-type formula ([6], [10]). See [10] for the proof of Stiefel-Whitney homology classes' version.

THEOREM 5.1. Let $\xi=(E, \iota, X)$ be an oriented block bundle over a compact $P L-p s e u d o-m a n i f o l d ~ X$ which can be stratified with only strata of even codimension. Then 


$$
\iota_{*} L_{*}(X)=\left(L_{*}(E) \cap U_{\xi}\right) \cap e^{*-1} \bar{l}(\xi) \text {. }
$$

Proof. Let $\bar{E}$ be the total space of the sphere bundle associated with $\xi$. Put $\tilde{E}=\operatorname{cl}(\partial E-\bar{E})$. Assume that $E$ is properly $P L$-embedded in $D^{\alpha}$ for $\alpha$ sufficiently large. Denote by $M$ a regular neighborhood of $E$ in $D^{\alpha}$. Let $\tilde{M}$ be a regular neighborhood of $\partial X$ in $\partial D^{\alpha}$ such that $\tilde{E}=\tilde{M} \cap E$. Put $\bar{M}=c l(\partial M-\tilde{M})$. Let $g: E \rightarrow M$ be the inclusion. Put $f=g \circ$. We will prove the following:

$$
\left\langle\varphi_{*}\left([V] \cap l^{*}(V)\right),([M] \cap)^{-1} g_{*}\left\{\left(L_{*}(E) \cap U_{\xi}\right) \cap \iota^{*-1} \bar{l}(\xi)\right\}\right\rangle=\sigma_{f}(\varphi, V)
$$

for $(\varphi, V)$ in $\Omega_{*}(M, \bar{M})$. Consequently, we obtain Theorem 5.1 from Corollary 4.4 and in view of the definition of $L_{*}(X)$ (cf. Definition 4.2). We can easily see that the left side of the identity is equal to that of the stable version. Then we may assume that $\varphi:(V, \partial V) \rightarrow(M, \bar{M})$ is a $P L$-embedding and $\varphi(V)$ is transverse to $X$ and $E$. Let $\nu=\left(N, \varphi_{N}, V\right)$ be a normal block bundle of $\varphi: V \rightarrow M$. Assume that $X$ and $E$ are transverse to $\nu$. Let $U_{\nu}$ be the Thom class of $\nu$. Then $[N] \cap U_{\nu}=\varphi_{N *}[V]$. Put $W=\left(L_{*}(E) \cap U_{\xi}\right) \cap e^{*-1} l(\xi)$. Then

$$
\begin{aligned}
& \left\langle\varphi_{*}\left([V] \cap l^{*}(V)\right),([M] \cap)^{-1} g_{*} W\right\rangle \\
& =\left\langle[V], l^{*}(V) \cup \varphi^{*}([M] \cap)^{-1} g_{*} W\right\rangle \\
& =\left\langle\varphi_{N *}^{-1}\left([N] \cap U_{\nu}\right), \varphi^{*}([M] \cap)^{-1} g_{*} W \cup l^{*}(V)\right\rangle \\
& =\left\langle[N], U_{\nu} \cup \varphi_{N}^{*-1} \varphi^{*}([M] \cap)^{-1} g_{*} W \cup \varphi_{N}^{*-1} l^{*}(V)\right\rangle .
\end{aligned}
$$

Note that $l^{*}(V)=\tilde{l}(\nu)$. Put $\varepsilon=(-1)^{\operatorname{codim} f \cdot \operatorname{codim} \varphi}$. Then

$$
\begin{aligned}
& \left\langle\varphi_{*}\left([V] \cap l^{*}(V),([M] \cap)^{-1} g_{*} W\right\rangle\right. \\
& =\varepsilon\left\langle[N] \cap \varphi_{N}^{*-1} \varphi^{*}([M] \cap)^{-1} g_{*} W, U_{\nu} \cup \varphi_{N}^{*-1} \bar{l}(\boldsymbol{\nu})\right\rangle .
\end{aligned}
$$

Let $\bar{N}$ be the total space of the sphere bundle associated with $\nu$. Put $\tilde{N}=c l(\partial N-\bar{N})$ and $\hat{M}=c l(M-N)$. Let $j_{N}:(N, \bar{N}, \tilde{N}) \rightarrow(M, \hat{M}, M)$ and $i_{M}:(M, \tilde{M})$ $\rightarrow(M, \hat{M})$ be the inclusions. Put $\hat{E}=\operatorname{cl}(E-N)$. Let $j_{E}:(E \cap N ; E \cap \bar{N}, \bar{E} \cap N)$ $\rightarrow(E ; \hat{E}, \bar{E}), i_{E}:(E, \tilde{E}) \rightarrow(E, \hat{E})$ and $g_{N}(E \cap N, E \cap \bar{N}) \rightarrow(N, \bar{N})$ be the inclusions. Then we have the following commutative diagram:

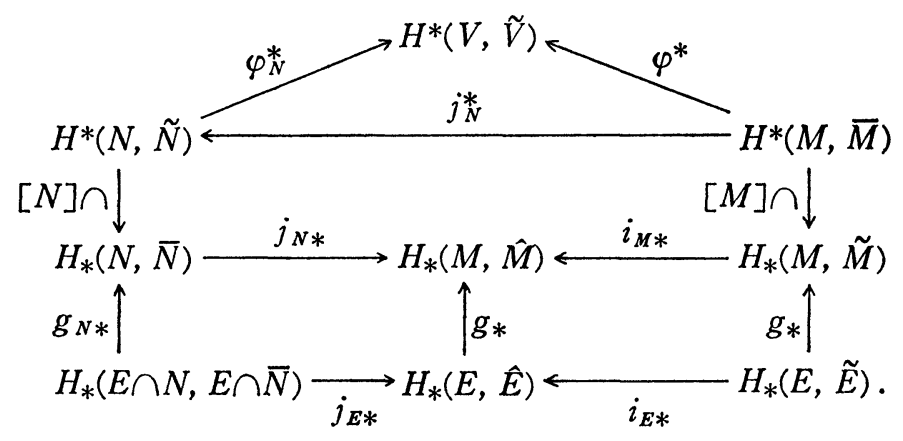


Note that $\varphi_{N}^{*}, j_{N *}$ and $j_{E *}$ are isomorphisms. Then

$$
\begin{aligned}
& {[N] \cap \varphi_{N}^{*-1} \varphi^{*}([M] \cap)^{-1} g_{*} W=j_{N *}^{-1} i_{M *} g_{*} W} \\
& =j_{N *}^{-1} i_{M *} g_{*}\left(L_{*}(E) \cap\left\{U_{\xi} \cup \iota^{*-1} \bar{l}(\xi)\right\}\right) \\
& =g_{N * j_{E *} i_{E *}}\left(L_{*}(E) \cap\left\{U_{\xi} \cup \iota^{*-1} \bar{l}(\xi)\right\}\right) \\
& =g_{N *}\left(j_{E *}^{-1} i_{E *} L_{*}(E) \cap j_{E}^{*}\left(U_{\xi} \cup \iota^{*-1} l(\xi)\right)\right) \text {. }
\end{aligned}
$$

By Axiom L2, we have $j_{E *}^{-1} i_{E *} L(E)=j_{E}^{\#} L_{*}(E)=L_{*}(E \cap N)$. Then

$$
\begin{aligned}
\left\langle\varphi_{*}(\right. & {\left.\left.[V] \cap l^{*}(V)\right),([M] \cap)^{-1} g_{*} W\right\rangle } \\
\quad & =\varepsilon\left\langle g_{N *}\left(L_{*}(E \cap N) \cap j_{E}^{*}\left(U_{\xi} \cup{\vartheta^{*-1}}^{*}(\xi)\right)\right), U_{\nu} \cup \varphi_{N}^{*-1} \bar{l}(V)\right\rangle \\
\quad & =\varepsilon\left\langle L_{*}(E \cap N), j_{E}^{*} U_{\xi} \cup g_{N}^{*} U_{\nu} \cup j_{E}^{*-1} \iota^{*-1} \bar{l}(\xi) \cup g_{N}^{*} \varphi_{N}^{*-1} \bar{l}(\nu)\right\rangle .
\end{aligned}
$$

Note that $j_{E}^{*} U_{\xi} \cup g_{N}^{*} U_{\nu}=U_{\xi|X \cap V \oplus \nu| X \cap V}$ and

$$
j_{E}^{*-1} \iota^{*-1} \bar{l}(\xi) \cup g_{N}^{*} \varphi_{N}^{*-1} \bar{l}(\nu)=\iota_{X}^{*} \cap V^{-1} \bar{l}(\xi|X \cap V \oplus \nu| X \cap V),
$$

where $\iota_{X \cap V}: X \cap V \rightarrow E \cap N$ is the inclusion. Then

$$
\begin{aligned}
& \left\langle\varphi_{*}\left([V] \cap l^{*}(V)\right),([M] \cap)^{-1} g_{*} W\right\rangle \\
& =\varepsilon\left\langle L_{*}(E \cap N), U_{\xi|X \cap V \oplus \nu| X \cap V} \cup_{X}^{*} \cap E^{-1} l(\xi|X \cap V \oplus \nu| X \cap V)\right\rangle .
\end{aligned}
$$

By Proposition 4.9, we have

$$
\begin{aligned}
\left\langle\varphi_{*}\left([V] \cap l^{*}(V)\right),([M] \cap)^{-1} g_{*} W\right\rangle & =\varepsilon \bar{\sigma}_{\xi|X \cap V \oplus \nu| X \cap V}(i d, E \cap N) \\
& =\varepsilon \sigma(X \cdot V)=\sigma(V \cdot X) .
\end{aligned}
$$

In view of the definition of $\sigma_{f}$, we have $\sigma(V \cdot X)=\sigma_{f}(\varphi, V)$. Then for each $(\varphi, V)$ in $\Omega_{*}(M, \bar{M})$, we have

$$
\left\langle\varphi_{*}\left([V] \cap l^{*}(V)\right),([M] \cap)^{-1} g_{*}\left(\left(L_{*}(E) \cap U_{\xi}\right) \cap c^{*-1} i(\xi)\right)\right\rangle=\sigma_{f}(\varphi, V) .
$$

By Corollary 4.4 and in view of the definition of $L(X)$ (cf. Definition 4.2), we have $g_{*}\left(\left(L_{*}(E) \cap U_{\xi}\right) \cap \iota^{*-1} \bar{l}(\xi)\right)=g_{* \iota_{*}} L_{*}(X)$. Then $\left(L_{*}(E) \cap U_{\xi}\right) \cap \iota^{*-1} \bar{l}(\xi)=\iota_{*} L_{*}(X)$.

Proof of theorem. The case where $X$ and $Y$ are collarable implies the general case. Thus we may suppose that $X$ and $Y$ are collarable in $M$. We will prove that

$$
\begin{array}{r}
\left\langle\varphi_{*}\left([V] \cap l^{*}(V)\right),([M] \cap)^{-1}\left\{\left(f_{*} L_{*}(X) \cdot g_{*} L_{*}(Y) \cap \bar{l}(M)\right) \cap \bar{l}(M)\right\rangle=\sigma_{h}(\varphi, V)\right. \\
\text { for each }(\varphi, V) \text { in } \Omega_{*}(M, \bar{M}) .
\end{array}
$$

This implies our theorem by Theorem 4.5.

Let $\varphi: V \rightarrow M$ be a map in $\Omega_{*}(M, \bar{M})$. We can choose a $P L$-embedding $\phi: V \rightarrow M \times D^{\alpha}$ for $\alpha$ sufficiently large such that $\psi$ is homotopic to $\varphi \times\{0\}$ : 
$V \rightarrow M \times D^{\alpha}$ and $\phi(V)$ is transverse to $(X \cup Y) \times D^{\alpha}$ in $M \times D^{\alpha}$. Hence we give the proof only when $\varphi: V \rightarrow M$ is a $P L$-embedding such that $\varphi(V)$ is transverse to $X \cup Y$ in $M$. We thus assume that $\varphi: V \rightarrow M$ is a $P L$-embedding with a normal bundle $\nu=\left(E, \varphi_{E}, V\right)$. We have the following commutative diagram:

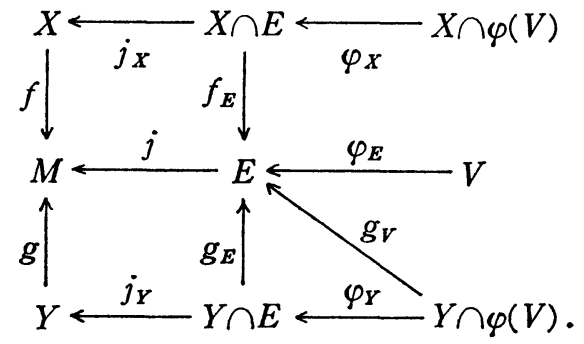

Here all maps except $\varphi_{E}: V \rightarrow E$ are inclusions and $\nu^{\prime \prime}=\left(X \cap E, \varphi_{X}, X \cap \varphi(V)\right)$ and $\nu^{\prime}=\left(Y \cap E, \varphi_{Y}, Y \cap \varphi(V)\right)$ are block bundles. Let $U_{\nu}$ be the Thom class of the normal block bundle $\nu=\left(E, \varphi_{E}, V\right)$, that is, $[E] \cap U_{\nu}=\varphi_{E *}[V]$. Let $\dot{l}(\nu)$ be the dual Hirzebruch $L$-homology class of the normal block bundle $\nu$. Note that $\bar{l}(\nu)=\varphi^{*} \bar{l}(M) \cup l^{*}(V)$ and $L_{*}(V)=[V] \cap l^{*}(V)$.

We put $W(f)=([M] \cap)^{-1} f_{*} L_{*}(X), W(g)=([M] \cap)^{-1} g_{*} L_{*}(Y)$ and

$$
P=\left\langle\varphi_{*}\left([V] \cap l^{*}(V)\right),([M] \cap)^{-1}\left\{\left(f_{*} L_{*}(X) \cdot g_{*} L_{*}(Y) \cap \bar{l}(M)\right) \cap \bar{l}(M)\right\}\right\rangle .
$$

Noting that $[E] \cap U_{\nu}=\varphi_{E *}[V]$, we have

$$
\begin{aligned}
P & =\left\langle[V] \cap l^{*}(V), \varphi^{*} W(f) \cup \varphi^{*} W(g) \cup \varphi^{*} \bar{l}(M)^{2}\right\rangle \\
& =\left\langle\varphi_{E *}^{-1}\left([E] \cap U_{\nu}\right), \varphi^{*} W(f) \cup \varphi^{*} W(g) \cup l^{*}(V) \cup \varphi^{*} \bar{l}(M)^{2}\right\rangle \\
& =\left\langle[E], U_{\nu} \cup \varphi_{E}^{*-1} \varphi^{*} W(f) \cup \varphi_{E}^{*-1} \varphi^{*} W(g) \cup \varphi_{E}^{*-1} l^{*}(V) \cup \varphi_{E}^{*-1} \varphi^{*} \bar{l}(M)^{2}\right\rangle .
\end{aligned}
$$

On the other hand, we have $[E] \cap \varphi_{E}^{*-1} \varphi^{*} W(f)=f_{E *} j_{X *}^{*} L_{*}(X)$ and $[E] \cap \varphi_{E}^{*-1} \varphi^{*} W(g)$ $=g_{E * j} j_{Y} L_{*}(Y)$. By Axiom L2, we have $j_{Y} L_{*}(X)=L_{*}(X \cap E)$ and $j_{Y}^{*} L_{*}(Y)=$ $L_{*}(Y \cap E)$. Furthermore we have $l^{*}(V) \cup \varphi^{*} \bar{l}(M)=\bar{l}(\nu)$ and $\varphi_{E}^{*-1} \varphi^{*}(\bar{l}(M)=\bar{l}(E)$. Put $\varepsilon=(-1)^{\operatorname{codim} f \cdot \operatorname{codim} \varphi}$. Then

$$
\begin{aligned}
P & =\varepsilon\left\langle f_{E *} L_{*}(X \cap E),([E] \cap)^{-1} g_{E *} L_{*}(Y \cap E) \cup U_{\nu} \cup \varphi_{E}^{*-1} \bar{l}(\nu) \cup \bar{l}(E)\right\rangle \\
& =\varepsilon\left\langle f_{E *} L_{*}(X \cap E),([E] \cap)^{-1} g_{E *}\left\{\left(L_{*}(Y \cap E) \cap g_{E}^{*} U_{\nu}\right) \cap g_{E}^{*} \varphi_{E}^{*-1} \bar{l}(\nu)\right\} \cup \bar{l}(E)\right\rangle .
\end{aligned}
$$

Note that $g_{E}^{*} U_{\nu}=U_{\nu^{\prime}}$ and $g_{E}^{*} \varphi_{E}^{*-1} \bar{l}(\boldsymbol{\nu})=\varphi_{Y}^{*-1} \bar{l}\left(\nu^{\prime}\right)$. By Theorem 5.1 , we have

Then

$$
\left(L_{*}(Y \cap E) \cap U_{\nu^{\prime}}\right) \cap \varphi_{Y}^{*-1} \bar{l}\left(\nu^{\prime}\right)=\varphi_{Y *} L_{*}(Y \cdot \varphi(V)) .
$$

$$
P=\varepsilon\left\langle f_{E *} L_{*}(X \cap E),([E] \cap)^{-1}\left(g_{E *} \varphi_{Y *} L_{*}(Y \cdot \varphi(V)) \cap \bar{l}(E)\right)\right\rangle .
$$

By Proposition 4.6, we have $P=\varepsilon \bar{\sigma}_{g_{V}}\left(f_{E}, X \cap E\right)$. By Lemma 2.3, we have $Y \cap \varphi(V)$ is transverse to $X \cap E$ in $E$. In view of the definition $\bar{\sigma}_{g_{V}}$, we see 
that

Then

$$
P=\varepsilon \sigma((X \cap E) \cdot(Y \cdot \varphi(V)))
$$

$$
P=\boldsymbol{\varepsilon} \sigma(X \cdot(Y \cdot \varphi(V)))=\sigma(\varphi(V) \cdot(X \cdot Y))=\sigma_{h}(\varphi, V) .
$$

Consequently, Theorem 4.5 implies that

$$
f_{*} L_{*}(X) \cdot g_{*} L_{*}(Y) \cap \bar{l}(M)=h_{*} L_{*}(X \cdot Y) . \quad \text { q. e. d. }
$$

\section{REFERENCES}

[1] E. AkIn, Stiefel-Whitney homology classes and cobordism, Trans. Amer. Math. Soc. 205 (1975), 341-359.

[2] G. A. Anderson, Resolution of generalized polyhedral manifolds, Tôhoku Math. J. 31 (1979), 495-517.

[3] M. A. ARmStrong, Transversality for polyhedra, Ann. of Math. 86 (1967), 172-191.

[4] S. Buoncristiano, C.R. Rourke and B. J. Sanderson, A geometric approach to homology theory, London Math. Soc. Lecture notes 18, 1976.

[5] A. Dold, Relations between ordinary and extraordinary homology, Colloq. on Alg. Top., Aarhus Univ., 1962, 2-9.

[6] W. Fulton and R. MAcPherson, Categorical framework for the study of singular spaces, Mem. Amer. Math. Soc. 243, 1981.

[7] M. Goresky AND R. MACPherson, Intersection homology theory, Topology 19 (1980), 135-162.

[8] M. Goresky And R. MACPherson, Intersection homology II, Invent. Math. 72 (1983), 77-129.

[9] A. MAtsui, Stiefel-Whitney homology classes of $\boldsymbol{Z}_{2}$-Poincaré-Euler spaces, Tôhoku Math. J. 35 (1983), 321-339.

[10] A. Matsui and H. Sato, Stiefel-Whitney homology classes and Riemann-Roch formula, In homotopy theory and related topics (H. Toda, ed.) Advanced Studies in Pure Math. 9 (1986), Kinokuniya, North-Holland, 129-134.

[11] A. Matsui, Intersection formula for Stiefel-Whitney homology classes, Tôhoku Math. J. 40 (1988), 315-322.

[12] C. MCCRory, Cone complexes and PL-transversality, Trans. of Amer. Math. Soc. 207 (1975), 269-291.

[13] J. Milnor And J. Stasheff, Characteristic classes, Ann. of Math. Studies 76, Princeton Univ. Press. 1974.

[14] C. R. Rourke and B. J. Sanderson, Block bundles I and II, Ann. of Math. 87 (1968), 1-28, 255-277.

[15] D. Sullivan, Combinatorial invariant of analytic spaces, Proc. Liverpool Singularities I, Lecture Notes in Math. 192, Springer-Verlag, Berlin-Heidelberg-New York, 1971, 165-168.

[16] D. Stone, Stratified polyhedra, Lecture Notes in Math. 252, Springer-Verlag, Berlin-Heidelberg-New York, 1972.

\footnotetext{
Department of Mathematics

FACULTY OF EDUCATION

FUKUSHIMA UNIVERSITY

FukUshima 960-12, JAPAN
} 